\title{
Melanocortin-4 Receptor Regulates Hippocampal Synaptic Plasticity through a Protein Kinase A-Dependent Mechanism
}

\author{
Yang Shen, Wing-Yu Fu, Elaine Y. L. Cheng, Amy K. Y. Fu, and Nancy Y. Ip \\ Division of Life Science, Molecular Neuroscience Center and State Key Laboratory of Molecular Neuroscience, The Hong Kong University of Science \\ and Technology, Clear Water Bay, Hong Kong, China
}

Learning and memory require orchestrated regulation of both structural and functional synaptic plasticity in the hippocampus. While a neuropeptide alpha-melanocyte-stimulating hormone, $\alpha$-MSH, has been implicated in memory acquisition and retention, the functional role of its cognate receptor, melanocortin-4 receptor (MC4R), in hippocampal-dependent synaptic plasticity has not been explored. In this study, we report that activation of MC4R enhances synaptic plasticity through the regulation of dendritic spine morphology and abundance of AMPA receptors. We show that activation of postsynaptic MC4R increases the number of mature dendritic spines and enhances surface expression of AMPA receptor subunit GluA1, resulting in synaptic accumulation of GluA1-containing AMPA receptors. Moreover, MC4R stimulates surface GluA1 trafficking through phosphorylation of GluA1 at Ser845 in a G $\alpha_{\mathrm{s}}$-cAMP/PKA-dependent manner. Blockade of protein kinase A (PKA) signaling abolishes the MC4R-mediated enhancement of neurotransmission and hippocampal long-term potentiation. Importantly, in vivo application of MC4R agonists increases LTP in the mouse hippocampal CA1 region. These findings reveal that MC4R in the hippocampus plays a critical role in the regulation of structural and functional plasticity.

\section{Introduction}

Synaptic plasticity, a key cellular mechanism that alters the efficacy of synaptic transmission between neurons in response to a new experience, is believed to underlie learning and memory. In particular, long-term potentiation (LTP) at hippocampal CA1 glutamatergic synapses is the most characterized form of synaptic plasticity. A myriad of concerted biochemical and cellular processes occur in response to increased neural activity during synaptic plasticity, including rapid changes in both the morphology and density of dendritic spines, abundance as well as subunit composition of AMPA-subtype glutamate receptors, regulation of local translation, and modulation of transcription through retrograde signaling to the nucleus (Earnshaw and Bressloff, 2006; Lee and Silva, 2009). Furthermore, long-term information can be stored in the brain not only through modulating synaptic strength, but also by the formation of new synapses or the elimination of existing synapses (Yuste and Bonhoeffer, 2001; Chklovskii, 2004; Fu et al., 2007; Fu et al., 2011). Importantly, abnormalities in synaptic plasticity are associated with impaired

Received July 10, 2012; revised 0ct. 8, 2012; accepted 0ct. 23, 2012.

Author contributions: Y.S., W.-Y.F., A.K.Y.F., and N.Y.I. designed research; Y.S. and E.Y.L.C. performed research; N.Y.I. contributed unpublished reagents/analytic tools; Y.S., W.-Y.F., A.K.Y.F., and N.Y.I. analyzed data; Y.S., A.K.Y.F., and N.Y.I. wrote the paper.

This study was supported in part by the Research Grants Council of Hong Kong Special Administrative Region (HKUST 661109, 661309, and 660810), the National Key Basic Research Program of China (973 Program) 2013CB530900, the S. H. Ho Foundation, and the Innovation and Technology Fund for State Key Laboratory (ITCPT/ 17-9). We thank Min Tian, Busma Butt, Cara Kwong, and William Chau for their excellent technical assistance and members of the Ip laboratory for many helpful discussions.

The authors declare no competing financial interests.

Correspondence should be addressed to Prof. Nancy Y. Ip at the above address. E-mail: BOIP@UST.HK.

DOI:10.1523/JNEUROSCI.3282-12.2013

Copyright $\odot 2013$ the authors $\quad 0270-6474 / 13 / 330464-09 \$ 15.00 / 0$ cognitive functions observed in various neurodegenerative diseases and psychiatric disorders (Cheung and Ip, 2011).

Synaptic plasticity can be regulated by various neuromodulators, including neurotransmitters (e.g., dopamine, serotonin, and norepinephrine) and neuropeptides (Yang, 2000; Murchison et al., 2004; O'Dell et al., 2010). Some of the unconventional neuropeptides include the regulators of food intake and energy metabolism, such as leptin and ghrelin (Gómez-Pinilla, 2008), and the central melanocortin system, which is fundamental in regulating body weight and energy homeostasis. The melanocortin neuropeptides, including $\alpha$-melanocyte-, $\beta$-melanocyte-, and $\gamma$-melanocytestimulating hormones (MSHs) and adrenocorticotropic hormones (ACTHs), are all derived from posttranslational processing of the precursor protein proopiomelanocortin (POMC). Interestingly, $\alpha$-MSH and MSH/ACTH analogs have long been implicated to facilitate learning and memory (Datta and King, 1982; McLay et al., 2001). However, the underlying mechanism remains largely unknown. Actions of melanocortins are mediated by the activation of melanocortin receptors, two of which, melanocortin-3 and melanocortin-4 receptor (MC4R), are predominantly expressed in the brain (Garfield et al., 2009). The detection of both MC4R and $\alpha$-MSH in rodent hippocampus raises the intriguing possibility that MC4R might mediate the action of $\alpha$-MSH in hippocampusdependent learning and memory (Gantz et al., 1993; Liu et al., 2003). This notion is further supported by a previous report that stimulation of MC4R leads to the activation of $\mathrm{G} \alpha_{\mathrm{s}}$-cAMP-PKA, a signaling cascade that is critical for specific forms of synaptic plasticity (Waltereit and Weller, 2003).

Here, we report that activation of postsynaptic MC4R in the hippocampus facilitates synaptic transmission and plasticity through enhancement of AMPA receptor-mediated neurotransmission and LTP at the Schaffer collateral-CA1 
(SC-CA1) pathway. Intriguingly, we found that MC4R is crucial for maintenance of mature dendritic spines in hippocampal pyramidal neurons, and stimulation of MC4R by its ligand(s) leads to protein kinase A (PKA)-dependent insertion of synaptic AMPA receptors. Our findings reveal a novel function of MC4R in hippocampal synaptic plasticity, which may contribute to hippocampus-dependent learning and memory.

\section{Materials and Methods}

Constructs, chemicals, antibodies, cultures, and animals. The expression constructs used include the following: full-length human MC4R (from Missouri S\&T cDNA Resource Center, Rolla, MO); full-length rat MC4R cDNA (from Dr. X.-Y. Lu, University of Texas Health Science Center, San Antonio, TX); short hairpin RNA (shRNA)-resistant expression constructs of human MC4R and its point mutants (D90N and I125K) in pIRES-DesRed2 vector; and full-length rat MC4R cDNA in pCMW-2bSEP vector [from Dr. Kenny Chung, The Hong Kong University of Science and Technology (HKUST), Hong Kong, China]; and pCI-SEPGluA1 was provided by Dr. Robert Malinow (Addgene, Cambridge, MA; plasmid 24000). The pSUPER-based shRNA construct for rat MC4R, with target sequence $5^{\prime}$-TCAGAAACCATCGTCATCA-3', was constructed. For the lentiviral pFUGW-based shRNA construct for mouse MC4R, the target sequence is 5'-GAACAAGAACCTGCACTCA-3'. The lentiviral constructs were packaged into a virus at the Gene Transfer Vector Core of the University of Iowa (Iowa City, IA). The antibodies used include the following: anti-MC4R ( $\mathrm{N}$ terminus; Alomone Labs); anti-pSer845 GluAl and anti-GluAl antibodies (PhosphoSolutions); anti-GluA1 (N terminus; Calbiochem); anti-pSer831 GluA1 (Millipore Bioscience Research Reagents). D-Tyrosine melanotan II (D-Tyr MTII) was purchased from Phoenix Pharmaceuticals, MTII from Tocris Bioscience, and H89 from Sigma or Tocris Bioscience.

HEK293T cells were transfected with expression constructs using Lipofectamine and Plus reagent (Invitrogen). Cultured hippocampal neurons were prepared from embryonic day 18-19 rat embryos. Hippocampal neurons at 18-22 DIV were subjected to agonist treatment, and neurons at 16-19 DIV were transfected with different plasmids plus GFP vector or SEP-GluA1 construct using calcium phosphate precipitation for 3 days (Kopec et al., 2006; Fu et al., 2007). All animal procedures were conducted in accordance with the Guidelines of the Animal Care Facility of HKUST and were approved by the Animal Ethics Committee at HKUST.

Real time-PCR. Adult mice were sacrificed and brain slices of $500 \mu \mathrm{m}$ were prepared using a vibrating tissue slicer (Thermo Scientific HM 650 $\mathrm{V})$. Tissues from the CA1 regions and non-CA1 regions of hippocampus, hypothalamus, and cortex were collected. Total RNA was prepared using RNeasy columns (Qiagen) according to the manufacturer's instructions. Single-stranded cDNA was synthesized using SuperScript II Reverse Transcriptase (Invitrogen) and random hexamers. Real-time PCR was performed with SYBR Green-based reagents (SYBR Green PCR kit; Qiagen) using a 7500 Fast Real-Time PCR System (Applied Biosystems). Endogenous gene expression was normalized against GAPDH. Data were obtained from three independent experiments.

Primers used for real-time PCR were as follows: mouse MC4R, $5^{\prime}$ GCGTTTCGAATGGGTCGGAAACCA- ${ }^{\prime}$ and $5^{\prime}$-CCGCAATGGA AAGCAGGCTGCAA-3'; mouse POMC, 5'-CTGGAGCAACCCGCC CAAGGA-3' and 5'-GCGCGTTCTTGATGATGGCGTTCT-3'; mouse AgRP, 5' -CTCGTTCTCCGCGTCGCTGTG-3' and 5' -ACCCAGCTTGCGGCAGTAGCA-3'; mouse Agouti, 5'-GGCTTCGATGAAGAAGGTGGCAAGG-3' and $5^{\prime}$-CACTGGCAGGAGGCGCACGG-3'; mouse GAPDH 5' ${ }^{\prime}$ TGCACCACCAACTGCTTAGC-3' and 5'-GCCATGGACTGTGGTCATGAG-3'.

Measurement of cAMP level. The intracellular cAMP level was determined by cAMP ELISA kits (GE Healthcare). In brief, neurons were preincubated with IBMX (1-methyl-3-isobutylxanthine) and then treated with D-Tyr MTII at indicated concentrations for $30 \mathrm{~min}$. Data were presented as mean \pm SEM (from three independent experiments in duplicate).

Western blot, cell-surface biotinylation, and immunocytochemical analysis. HEK293T cells and cultured hippocampal neurons were lysed in radioimmunoprecipitation assay buffer, and mouse brains were homog- enized in lysis buffer as described (Fu et al., 2007). For surface biotinylation using hippocampal neurons, the procedure was performed as described previously (Fu et al., 2011). For surface staining of GluA1, hippocampal neurons at 22 DIV were incubated with antibodies against the extracellular domain of GluA1 at $37^{\circ} \mathrm{C}$ for $10 \mathrm{~min}$ (Fu et al., 2011). For virus infection, mouse brains were fixed with $4 \%$ paraformaldehyde for 1 day. Sections of $50 \mu \mathrm{m}$ were prepared using a vibrating tissue slicer (VT 1000S, Leica). Images were acquired using confocal microscope LSM-710 (Carl Zeiss).

Electrophysiology and stereotaxic surgery. Miniature excitatory postsynaptic current (mEPSC) was measured in rat hippocampal neurons at 18-22 DIV as described (Fu et al., 2011). For mEPSC recording, highdensity hippocampal cultures $\left(1.5 \times 10^{5}\right.$ cells/well in 12 -well plates $)$ were used for MC4R knockdown, whereas low-density hippocampal cultures $\left(0.5 \times 10^{5}\right.$ cells/well in 12 -well plates $)$ were used with agonist treatment. For LTP recordings, mouse brains were immediately dissected after sacrifice and soaked in ice-cold, $95 \% \mathrm{O}_{2} / 5 \% \mathrm{CO}_{2}$ oxygenated artificial cerebrospinal fluid. Brain slices at $300 \mu \mathrm{m}$ were prepared using a vibrating tissue slicer (Thermo Scientific HM650V), and field excitatory postsynaptic potentials (fEPSPs) were recorded using a MED64 multichannel recording system (Alpha MED Scientific). For each slice, baseline stimulus intensity was set at the level that elicited $\sim 40 \%$ of the maximum fEPSP response. LTP was induced with a conditioning stimulus consisting of three theta burst trains (ten $5 \mathrm{~Hz}$ series of four $100 \mathrm{~Hz}$ pulses for each, $40 \mathrm{~s}$ apart). At least eight slices from 3-4 mice for each condition were used.

Vesicular stomatitis virus glycoprotein G-pseudotyped lentiviral particles with pFUGW-shMC4R were delivered into mouse hippocampal CA1 region (anteroposterior, $-2.00 \mathrm{~mm}$; mediolateral, $\pm 1.50 \mathrm{~mm}$; dorsoventral, $-1.5 \mathrm{~mm}$; relative to bregma) for 3 weeks. Lentivirus injection was conducted using an animal stereotactic apparatus (Stoelting) and the Quintessential Stereotaxic Injector (catalog no. 53311, Stoelting) with an injection volume of $2 \mu \mathrm{l}$ at $0.2 \mu \mathrm{l} / \mathrm{min}$ connected to a customized borosilicate glass microcapillary tip of $\sim 100 \mu \mathrm{m}$.

Quantification and statistical analysis. Images were quantified in a double-blinded manner as described (Fu et al., 2007). A stack of images ( $z$ step, $0.4-0.5 \mu \mathrm{m}$ ) of $8-12$ layers was collected using a $60 \times$ objective. For each condition, $>15$ neurons from three independent experiments were analyzed using MetaMorph software (Universal Imaging). The spine length and volume were quantified as described (Schratt et al., 2006). For SEP-GluA1 quantification, the images were thresholded to exclude the background noise. The number of GFP-positive spines were then counted using MetaMorph software. For 3D reconstruction, filament tracing was performed with Imaris 7.2 (Bitplane). Statistical analysis was performed using Student's $t$ test and two-way or one-way ANOVA followed by Newman-Keuls test or Kruskal-Wallis test (when the data were not normally distributed). All experiments were performed at least three times, except for those specifically indicated.

\section{Results \\ MC4R is concentrated at postsynaptic regions in hippocampus}

We first examined the expression of MC4R and its ligands in rodent hippocampus. MC4R mRNA was prominent in the adult mouse hippocampus at a level similar to that in the hypothalamus. This was accompanied by a detectable level of mRNAs of MC4R ligands, including POMC, precursor protein of MC4R agonist $\alpha-\mathrm{MSH}$, and antagonists of MC4R (Agouti and Agoutirelated peptide, AgRP; Fig. 1a). Interestingly, endogenous MC4Rs were detected as surface clusters along dendritic shafts, with some clusters concentrated in dendritic spines of hippocampal neurons $(>21$ DIV; Fig. 1b). To confirm the synaptic localization of MC4R, cultured hippocampal neurons were transfected with a MC4R construct containing the $\mathrm{pH}$-sensitive GFP superecliptic pHluorin (SEP) tag at the extracellular N terminus (SEP-MC4R), which allows the visualization of MC4Rs upon incorporation into the cell membrane (where $\mathrm{pH}$ is $>7$ ). Consistent with the 

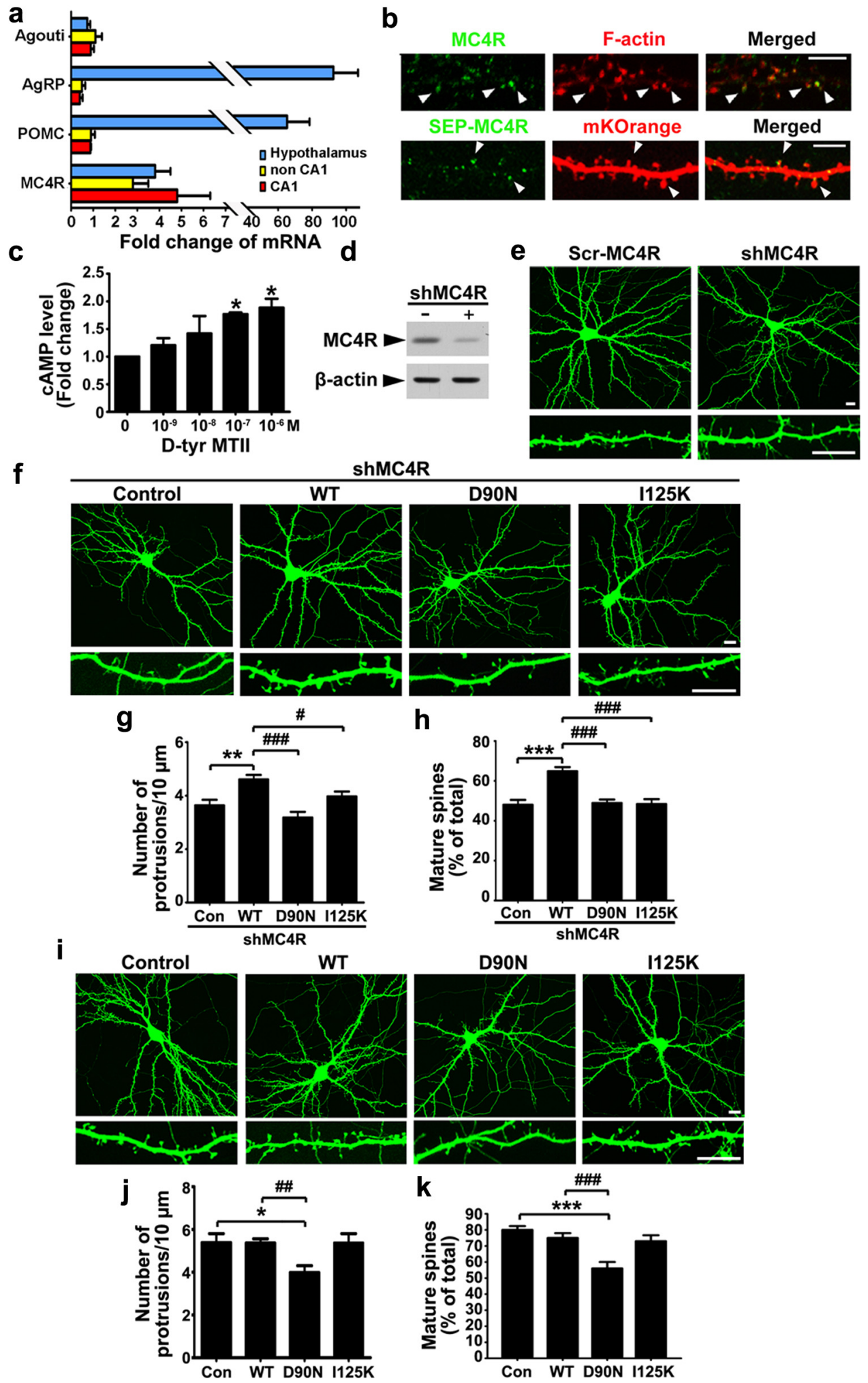

Figure 1. MC4Ractivation is required for the maintenance of mature spines and functional synapses. $\boldsymbol{a}$, Expression of MC4Rand itsligands in adultmouse hippocampus. mRNAs of different brain parts were prepared and subjected to real time-PCR. The data were presented as relative ratio of mRNA against that of cerebral cortex (agouti was used as a negative control). $\boldsymbol{b}$, Top row, Staining of surface MC4R in hippocampal neurons at 22 DIV. F-actin was labeled with rhodamine-phalloidin. Scale bar, $5 \mu \mathrm{m}$. Bottom row, cultured hippocampal neurons (17 DIV) were transfected with SEP-MC4R and mKOrange constructs, and were examined at 21 DIV. Scale bar, 10

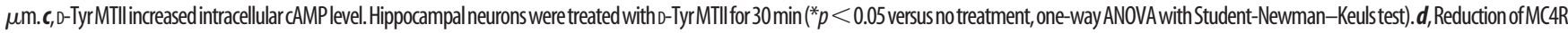
protein in HEK293T cells upon shMC4R knockdown (by $\sim 83.0 \pm 4.2 \% ; * * * 0<0.001, n=3$ experiments, Student'sttest). The cellswere cotransfected with EGFP-tagged rat MC4Rexpression construct, together witheither shMC4R(+) orpSUPERvector(-).e, Cultured hippocampal neuronswere cotransfected withshMC4Roritsscrambled shRNA(Scr-MC4R) with GFP plasmid. $\boldsymbol{f}$ - $\boldsymbol{h}$, Hippocampal neuronswere cotransfected with shMC4R and constructs expressing shRNA-resistanthuman MC4R(WT) or its pointmutants (D90N or I125K).f, Representativeimages. $\boldsymbol{g}, \boldsymbol{h}$, Quantification of spinedensity $(\boldsymbol{g})$ and the percentage of maturespines (spines with a mushroom head) (h). Data were expressed as mean \pm SEM; ${ }^{* *} p<0.01$ and ${ }^{* * *} p<0.001$ versus shMC4R; $\# p<0.05$ and \#\# $<0.001$ versus shMC4R+WT (one-way ANOVA with Student-Newman-Keuls test). $\boldsymbol{i}-\boldsymbol{k}, 0$ verexpression of hMC4R-WT and its point mutants (D90N or I250K) in cultured hippocampal neurons.i, Representative images.j, $\boldsymbol{k}$, Quantification of spine density (l) and percentage of mature spines $(\boldsymbol{k}){ }^{*} p<0.05$ and ${ }^{* * *} p<0.001$ versus control (Con); $\#^{\prime \prime}<0.01$ and ${ }^{\# \# \#} p<0.001$ versus WT (one-way ANOVA with Student-Newman-Keuls test). Scale bars, $10 \mu \mathrm{m}$. 
a

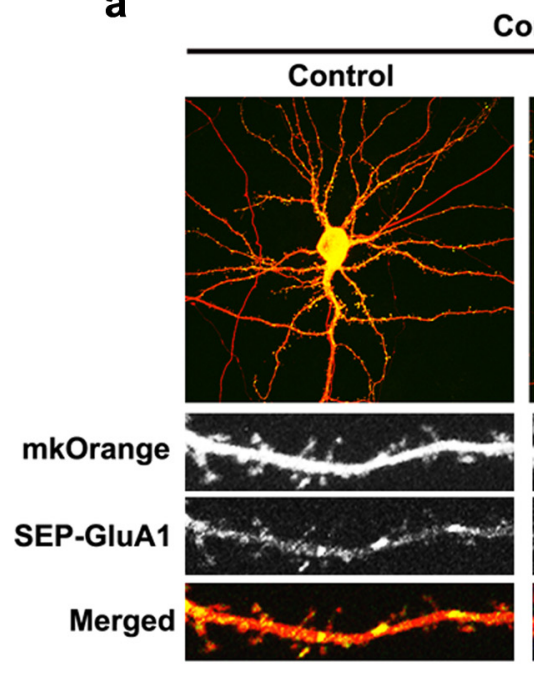

Con
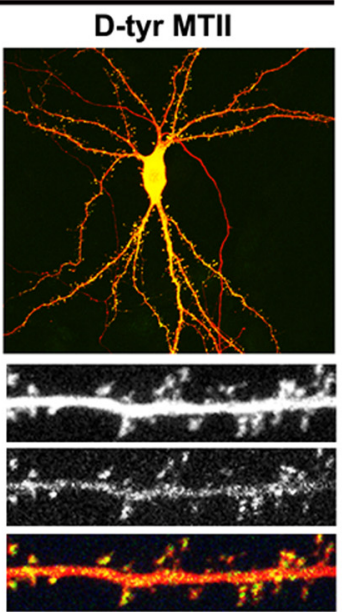

b

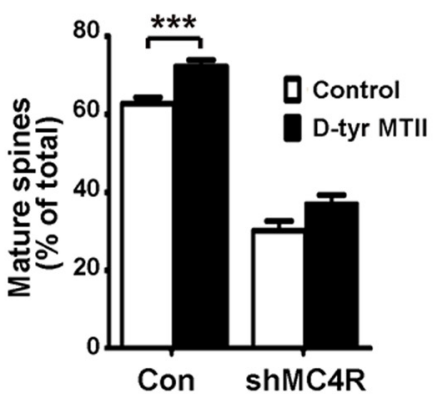

d
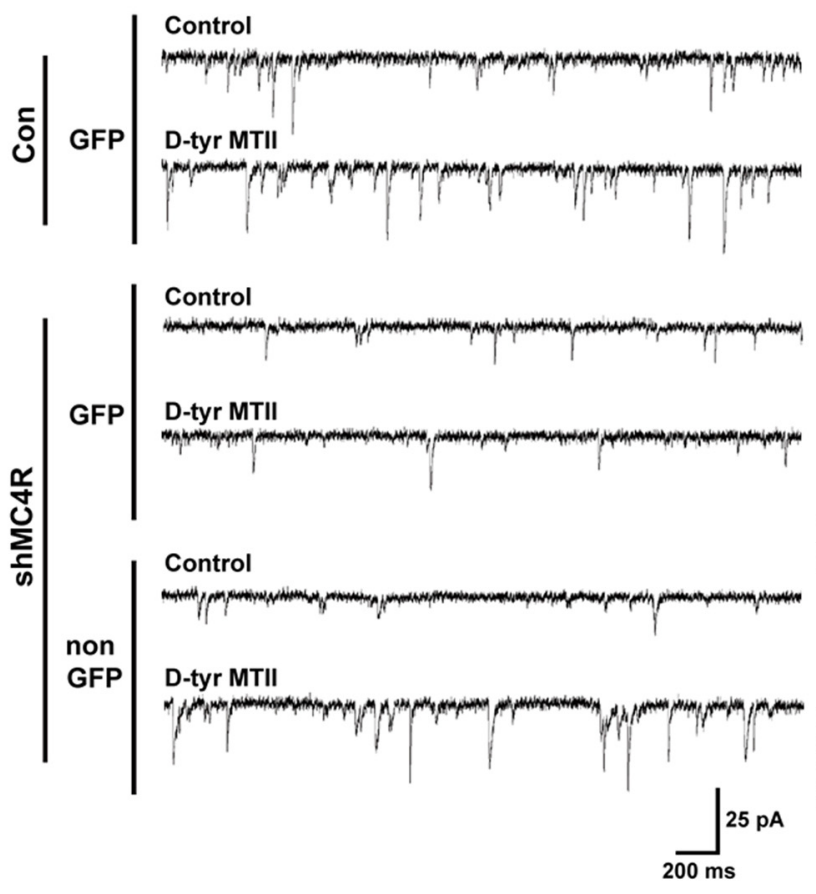

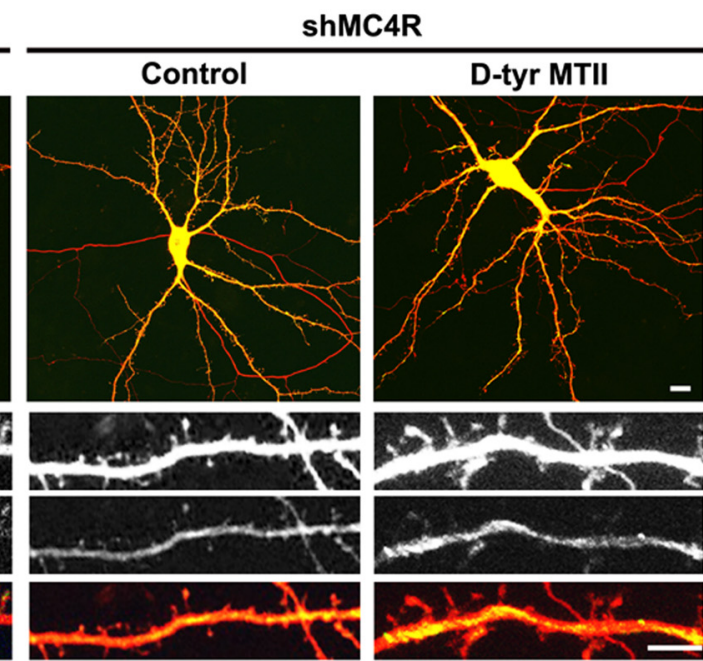

C

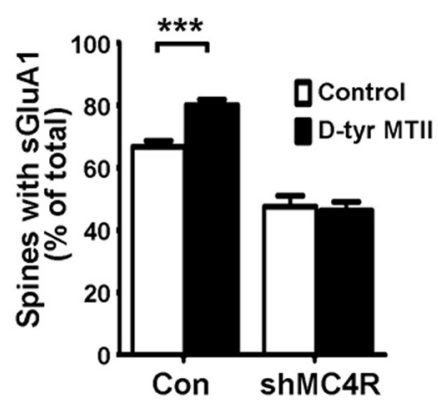

e
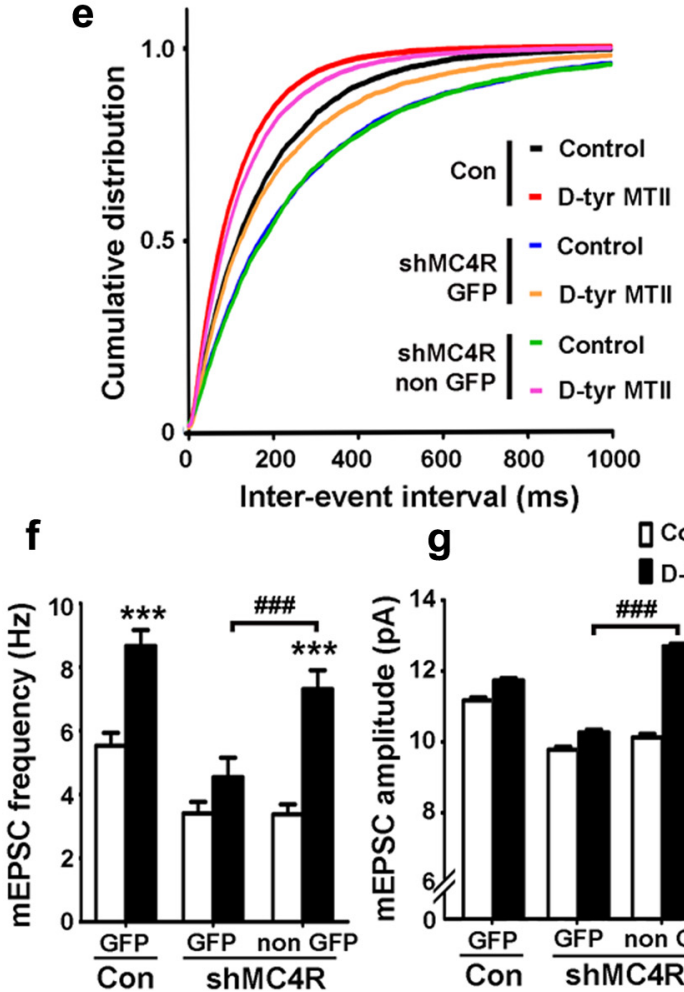

Figure 2. Activation of endogenous MC4Renhances maturation offunctional synapses and neurotransmission. $\boldsymbol{a}$ - $\boldsymbol{c}$, Silencing of MC4Rabolished the increase of mature spines and GluA1-containing spines by D-Tyr MTII $(1 \mu \mathrm{m}$ ). Hippocampal neurons were cotransfected with GFP plasmid togetherwithshMC4RandmKOrangeconstructsat 17-18DIVasindicated. Theneuronsat21-22DIV weretreated witho-TyrMTIIfor 2h. $\boldsymbol{a}$, Representative images. Scale bars: top row, $10 \mu \mathrm{m}$; bottom rows, $5 \mu \mathrm{m}$. $\boldsymbol{b}, \boldsymbol{c}$, Quantification of mature spines (b) and SEP-GluA1-containing spines (c). Data were expressed as mean \pm SEM; *** $p<0.001$, o-Tyr MTIl versus control (Con), two-way ANOVA. $\boldsymbol{d}-\boldsymbol{g}$, MC4R knockdown abolished the D-Tyr MTII-stimulated increase in neurotransmission. Hippocampal neurons were cotransfected with GFP construct together with orwithoutshMC4Rand then treated with D-Tyr MTII. $\boldsymbol{d}$, Representative mEPSC traces. $\boldsymbol{e}-\boldsymbol{g}$, Cumulative distribution of interevent intervals (inversely proportional to frequency $(\boldsymbol{e})$, and quantification of frequency $(\boldsymbol{f})$, and amplitude $(\boldsymbol{g})$ of $\mathrm{mEPSC}$. GFP-expressing neurons in the control, the shMC4R-transfected conditions, and theirneighboring untransfected neurons (non GFP) were recorded. Data were presented as mean \pm SEM; *** $p<0.001$, D-Tyr MTII versus Control treatment, two-way ANOVA; ${ }^{\# \#} p<0.001$ versus GFP cells in shMC4R (with D-Tyr MTIl treatment), one-way ANOVA with Student-Newman-Keuls test $(\boldsymbol{f})$; one-way ANOVA with Kruskal-Wallis test (g). 
a
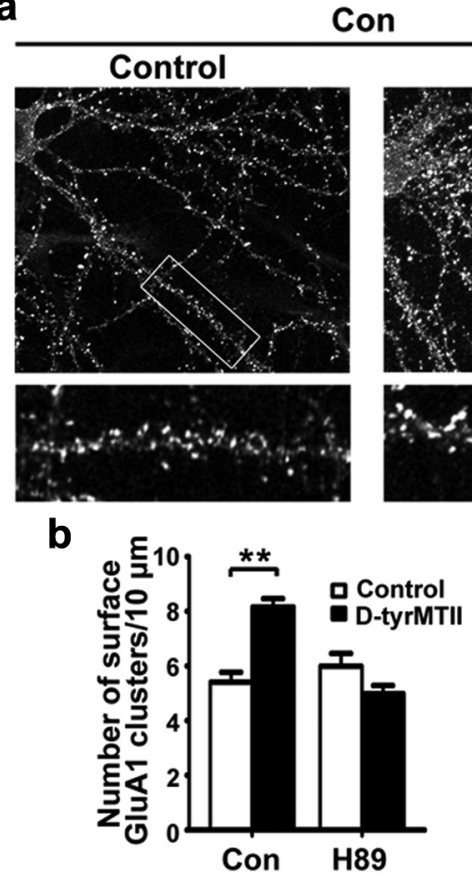

e

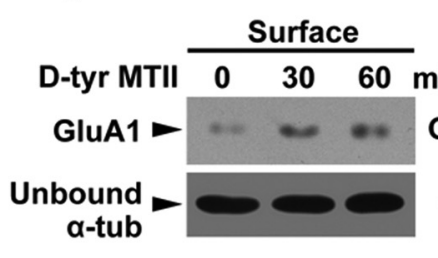

C
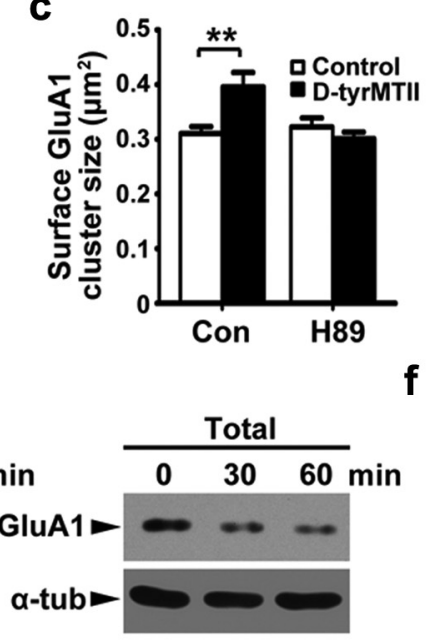

H89
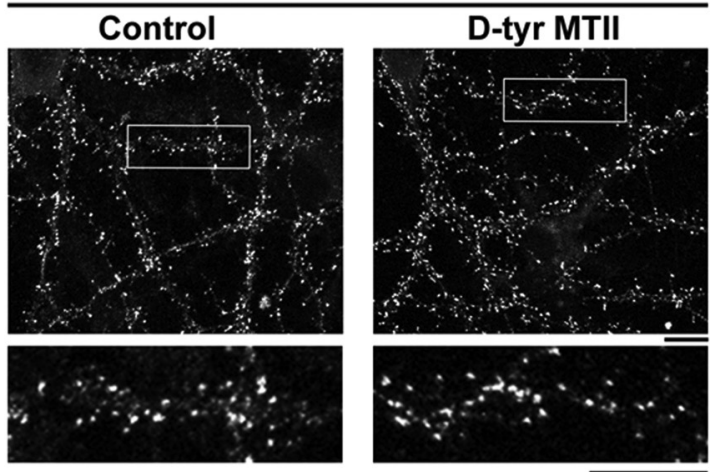

d

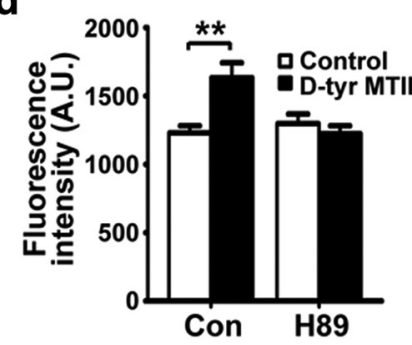

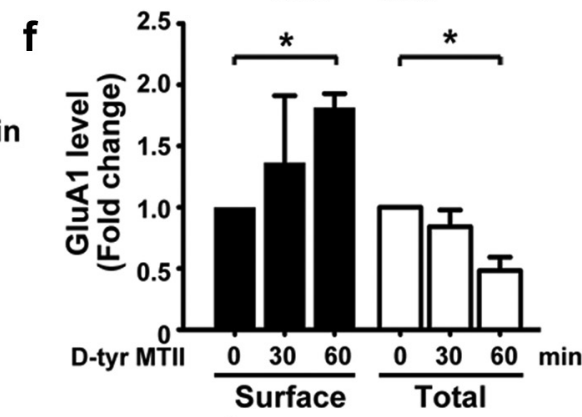
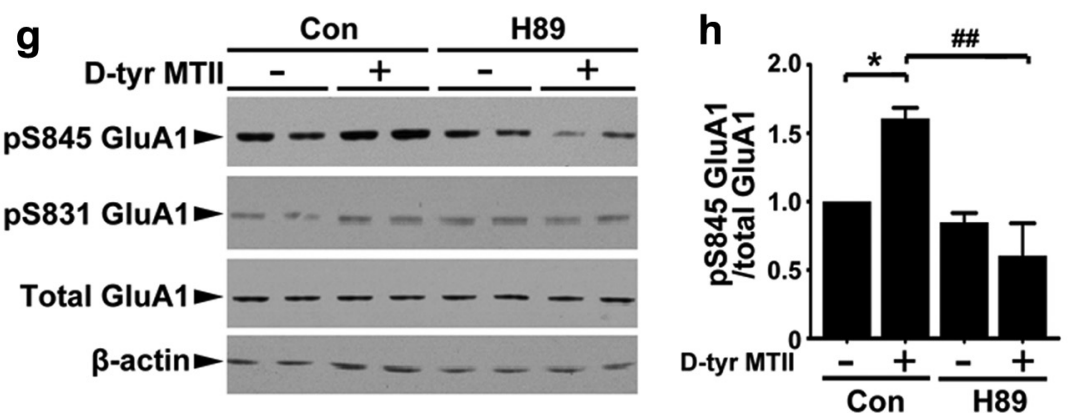

i

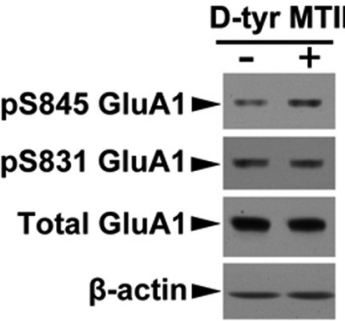

Figure 3. MC4R regulates surface levels of GluA1 through PKA-dependent phosphorylation. $\boldsymbol{a}-\boldsymbol{d}$, Hippocampal neurons were incubated with D-Tyr MTII (100 nM) and H89 (10 $\mu \mathrm{m})$ for $2 \mathrm{~h}$ and then stained for surface GluA1. $\boldsymbol{a}$, Representative images. Scale bar, $10 \mu \mathrm{m} . \boldsymbol{b}-\boldsymbol{d}$, Quantification of density (b), size (c), and intensity $(\boldsymbol{d})$ of surface GluA1 clusters. Data were expressed as mean \pm SEM, ${ }^{* *} p<0.01$, D-Tyr MTII versus control (two-way ANOVA); $n=10$ neurons from each experiment, two experiments). $\boldsymbol{e}$, Surface and total proteins of hippocampal neurons after D-Tyr MTII treatment were collected and subjected to Western blot analysis for GluA1.f, Fold change (three experiments; ${ }^{*} p<0.05$ versus 0 min, one-way ANOVA with Student-Newman-Keuls test. $\boldsymbol{g}$, $\boldsymbol{h}$, Hippocampal neurons were cotreated with D-Tyr MTII and H89 for $1 \mathbf{h}$. $g$, Western blot analysis. $\boldsymbol{h}$, Quantitative analysis; ${ }^{*} p<0.05$ d-Tyr MTII (+) versus no treatment $(-)$, two-way ANOVA; ${ }^{\# \#} p<0.01$, versus D-Tyr MTII, one-way ANOVA with Student-Newman-Keuls test. $i$, D-Tyr MTIl increased level of pSer845 GluA1 in cultured hippocampal slices (7 DIV).

endogenous immunostaining data, SEP-MC4R showed a similar spatial distribution pattern and could be detected on both dendritic shaft and dendritic spines (Fig. 1b). Together, these findings demonstrate that MC4R is localized to the postsynaptic sites of hippocampal neurons. Treatment of cultured hippocampal neurons with a selective MC4R agonist, D-Tyr MTII (a potent and stable cyclic analog of $\alpha-\mathrm{MSH}$ ), increased intracellular cAMP level in a dose-dependent manner (Fig. 1c) consistent with the notion that MC4R is a $\mathrm{G} \alpha_{\mathrm{s}}$-coupled $\mathrm{G}$ protein-coupled receptor (GPCR) (Oosterom et al., 2001).
MC4R activation is critical for maintaining functional synapses

In the mammalian brain, dendritic spines are the postsynaptic sites where excitatory synapses reside (Segal, 2005). Changes in the morphology and number of dendritic spines are closely associated with the regulation of synaptic strength (Yuste and Bonhoeffer, 2001). In cultured hippocampal neurons ( $>21$ DIV), the majority of dendritic spines adopt a mushroom-shaped structure that are enriched with postsynaptic proteins such as PSD-95 and excitatory glutamate receptors (Ethell and Pasquale, 2005). We 


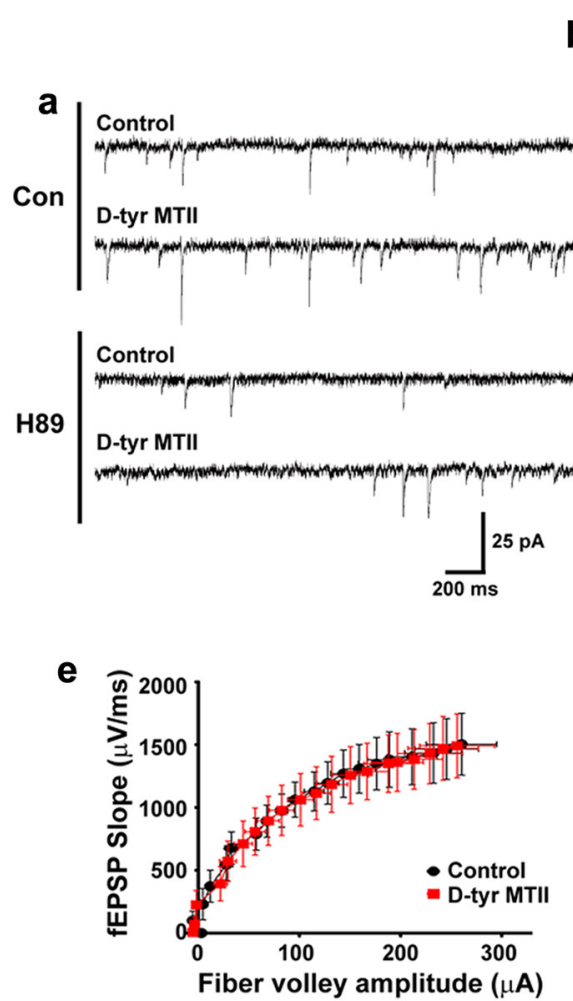

b

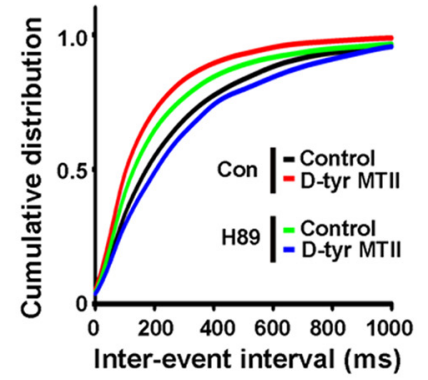

C

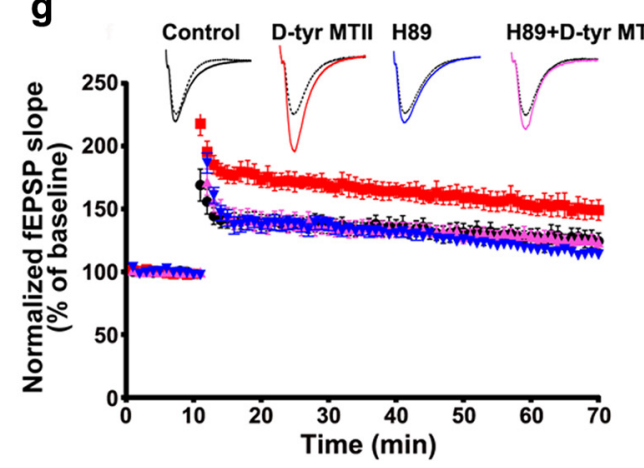

Figure 4. MC4R activation increases basal neurotransmission and LTP induction through PKA activation. $\boldsymbol{a}-\boldsymbol{d}$, Inhibition of PKA abolished the D-Tyr MTII-stimulated increase in basal neurotransmission. Hippocampal neurons were incubated with D-Tyr MTII $(100 \mathrm{~nm})$ and $\mathrm{H} 89(10 \mu \mathrm{m})$ for 2 h. $\boldsymbol{a}$, Representative mEPSC traces. $\boldsymbol{b}$ - $\boldsymbol{d}$, Cumulative distribution of interevent intervals $(\boldsymbol{b})$ quantification of frequency $(\boldsymbol{c})$, and amplitude $(\boldsymbol{d})$ of mEPSCS. Data were presented as mean \pm SEM, ${ }^{*} p<0.05, \mathrm{D}$-Tyr MTII versus control (Con), two-way ANOVA (c); ${ }^{* *} p<0.001$ versus control, one-way ANOVA with Kruskal-Wallis test (d). $\boldsymbol{e}-\boldsymbol{h}$, D-Tyr MTII enhanced LTP through activation of PKA. $\boldsymbol{e}, \boldsymbol{f}$, Basal synaptic transmission and short-term forms of synaptic plasticity were not altered by D-Tyr MTII treatment. $\boldsymbol{e}$, Input- output curve generated from the slope fEPSP versus fiber volley amplitude. $\boldsymbol{F}$, Paired pulse facilitation. Second stimuli were delivered at intervals as indicated. Percent facilitation of fEPSP slope of second response as percentage of first response. $\boldsymbol{g}, \boldsymbol{h}$, Acute hippocampal slices were treated with D-Tyr MTII (1 $\mu \mathrm{M})$ and H89 (20 $\mu \mathrm{m})$ for 2 h. $\boldsymbol{g}$, Summary plot of normalized fEPSP slope measurement (mean \pm SEM). $\boldsymbol{h}$, Quantification of fEPSP slope increase at $1 \mathrm{~h}$ after LTP induction (mean $\pm \mathrm{SEM}$; ${ }^{* *} p<0.01 \mathrm{D}$-Tyr MTII $(+)$ versus no treatment $(-)$, two-way ANOVA).

found that knockdown of MC4R using a MC4R shRNA construct (shMC4R) resulted in a reduction of dendritic spine density (by $\sim 30 \%$; Scr-MC4R, $5.45 \pm 0.17 / 10 \mu \mathrm{m}$; shMC4R, $3.63 \pm 0.21 / 10$ $\mu \mathrm{m} ; p<0.001$, Student's $t$ test) accompanied by a significant decrease (by $\sim 50 \%$ ) in the percentage of mature spines (spines with a mushroom head; Scr-MC4R, $62.8 \pm 1.5 \%$; shMC4R, $30.2 \pm 2.4 \% ; p<0.001$, Student's $t$ test; Fig. $1 d, e)$.

Reexpression of shRNA-resistant human MC4R in MC4Rknockdown neurons rescued the loss of mature spines. In contrast, coexpression of the MC4R point mutant D90N, which fails to bind $\mathrm{G} \alpha$, or $1125 \mathrm{~K}$, which cannot interact with endogenous agonist, could not restore the dendritic spine phenotype in
MC4R-knockdown neurons (Fig. $1 f-h$ ). Thus, both the stimulation of MC4R by its agonist as well as activation of the downstream $G$ protein-mediated signaling is essential for MC4R-mediated maintenance of mature spines. Interestingly, although ectopic expression of human MC4R wild type (WT) or I125K mutant did not regulate spine morphology nor their number, overexpression of $\mathrm{D} 90 \mathrm{~N}$ mutant reduced spine density (by 30\%) and elicited a substantial decrease in the percentage of mature spines (by $\sim 31 \%$; Fig. $1 i-k)$. The D90N mutant might therefore act in a dominant-negative manner by preventing the activation of endogenous MC4R and its effect on dendritic spines.

\section{Activation of MC4R enhances neurotransmission}

Next, we asked whether activation of endogenous MC4R enhances the number of GluA1-containing spines. We found that treatment of neurons with the MC4R agonist D-Tyr MTII not only enhanced the number of mature spines (by $~ 16 \%$ ), but also increased the proportion of spines that contained AMPA receptor subunit GluA1 (Fig. $2 a-c$ ). Intriguingly, knockdown of MC4R abolished the D-Tyr MTII-stimulated increase in mature spines, although a reduction of mature spines and GluA1-containing spines was also observed in the MC4R-depleted neurons (Fig. $2 a-c$ ).

Given the critical role of surface trafficking and synaptic expression of AMPA in synaptic transmission, we investigated how endogenous MC4R regulates neurotransmission. D-Tyr MTII treatment enhanced the frequency (with a reduction in interevent interval) accompanied by a slight increase in amplitude of AMPA receptor-mediated mEPSCs (Fig. $2 d-g$ ). Knockdown of MC4R in neurons (expressing with GFP) specifically abolished the D-Tyr MTII-mediated increase in mEPSC frequency and amplitude, whereas a similar reduction was not observed in the neighboring (non-GFP expressing) neurons or in the control GFP neurons (Fig. $2 d-g$ ). These findings indicate that activation of endogenous MC4R signaling enhances AMPA receptor-mediated synaptic transmission. Notably, reduced basal neurotransmission was observed in both MC4R-knockdown and its neighboring neurons, suggesting that neuronal connectivity is affected by the MC4R knockdownmediated postsynaptic changes (Fig. $2 d-g$ ).

\section{MC4R regulates surface level of GluA1 through PKA- dependent phosphorylation}

Surface trafficking of GluA1-containing AMPA receptor is regulated by PKA-dependent phosphorylation of GluA1 (Esteban et 
al., 2003). Together with the ability of MC4R to activate $\mathrm{G} \alpha_{\mathrm{s}}$-cAMP-PKA signaling (Man et al., 2007), this prompted us to ask whether PKA mediates the effect of MC4R on the regulation of synaptic GluA1. D-Tyr MTII increased the density (by $\sim 30 \%$ ), size (by $\sim 30 \%$ ), and fluorescence intensity (by $\sim 40 \%$ ) of surface GluA1 immunostaining in hippocampal neurons (Fig. $3 a-d$ ). We found that treatment of hippocampal neurons with PKA inhibitor $\mathrm{H} 89$ abolished the increase in surface GluA1 clusters induced by D-Tyr MTII (Fig. 3a-d), indicating that MC4R increases surface expression of GluA1 through the activation of $\mathrm{G} \alpha_{\mathrm{s}}$-cAMPPKA signaling. Using surface biotinylation assay, we confirmed that surface expression of GluA1 was enhanced upon MC4R activation and may therefore promote synaptic localization of GluA1-containing receptors (Fig. 3e,f). Phosphorylation of GluA1 at Ser845 promotes the surface delivery and synaptic targeting of GluA1-containing AMPA receptors (Derkach et al., 2007; Man et al., 2007). Supporting the notion that the $\mathrm{G} \alpha_{\mathrm{s}}$-cAMP-PKA signaling pathway mediates the observed MC4R-dependent changes in GluA1 localization, D-Tyr MTII significantly increased the phosphorylation of GluA1 at Ser845 in cultured hippocampal neurons (by $\sim 50 \%$ ), which was blocked by $\mathrm{H} 89$ co-treatment (Fig. $3 g, h$ ). In contrast, the CaMKII-dependent phosphorylation of GluA1 at Ser831 remained unchanged (Fig. $3 g, h$ ). Similarly, treatment of cultured hippocampal slices with D-Tyr MTII enhanced the phosphorylation of GluA1 at Ser845 but not Ser831 (Fig. 3i).

\section{MC4R activation enhances LTP through PKA activation}

Next, we examined whether PKA signaling mediates the MC4Rstimulated synaptic transmission and plasticity. While treatment with D-Tyr MTII led to obvious increases in both the frequency and amplitude of mEPSC, the induction was blocked by the cotreatment with H89 (Fig. 4a-d), suggesting that activation of MC4R increases AMPA receptor-mediated synaptic transmission in a PKA-dependent manner.

LTP is a major form of synaptic plasticity in the hippocampus that involves regulation of AMPA receptor trafficking. We therefore investigated the role of MC4R on LTP at the SC-CA1 synapses. D-Tyr MTII did not affect basal synaptic transmission (Fig. $4 e$ ) or presynaptic release as revealed by paired-pulse facilitation (Fig. $4 \mathrm{f}$ ). Using a stimulus similar to the physiological hippocampal activity, theta-burst stimulation (TBS), the magnitude of SCCA1 LTP exhibited a drastic increase upon D-Tyr MTII treatment (by $\sim 100 \%$ ). This induction was abolished in the presence of H89 (Fig. 4g,h). Thus, MC4R activation promotes the induction of hippocampal LTP, underscoring the importance of the MC4R signaling pathway in synaptic plasticity.

\section{Activation of MC4R regulates spine morphogenesis and LTP in vivo}

We then asked whether MC4R is important for regulating synaptic plasticity in vivo. Expression of MC4R in CA1 of mouse hippocampus was silenced by lentiviral-based MC4R shRNA. b

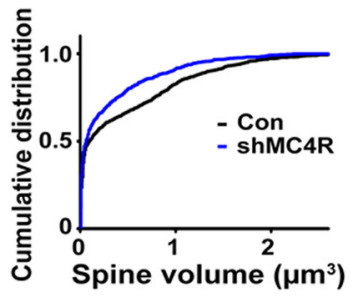

C

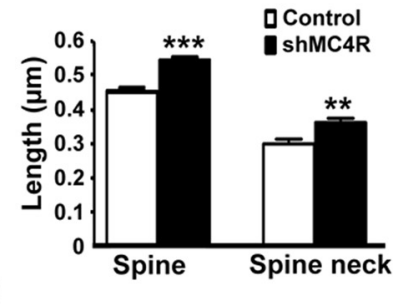

e
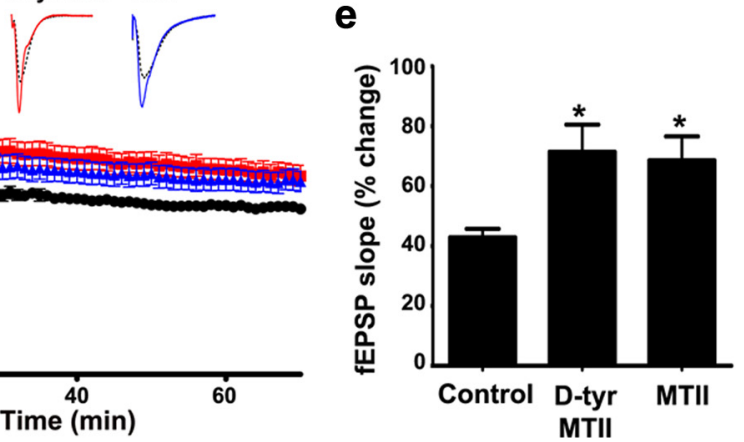

Figure 5. MC4R activation is important for maintaining dendritic spine morphology and enhancement of LTP in vivo. $\boldsymbol{a}-\boldsymbol{c}$, LTP in mouse CA1 region. $d$, Plots of normalized fEPSP slope measurement (mean \pm SEM). e, Quantification of fEPSP slope increase Control D-tyr MTII MTII
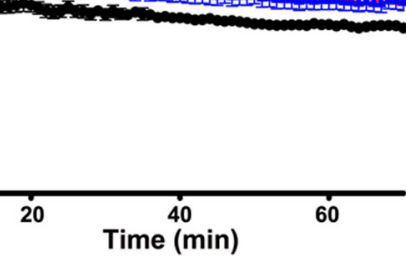
dent-Newman-Keuls test).

Consistent with the effect of MC4R knockdown in cultured hippocampal neurons, we found that MC4R-knockdown neurons in the CA1 region of the hippocampus showed reduced dendritic spine volume accompanied by increased proportion of immature spines (Fig. $5 a-c$ ). Since the size of spine heads is strongly correlated with synaptic strength, the reduction of dendritic spine volume may lead to weakening of existing synapses. We next examined whether in vivo delivery of MC4R agonists (D-Tyr MTII or MTII) through intraperitoneal injection affects LTP induction in hippocampal slice preparations. We found that peripheral administration of D-Tyr MTII and MTII substantially increased the magnitude of SC-CA1 LTP after TBS (both by $\sim 100 \%$; Fig. $5 d, e$ ). These results indicate that activation of MC4R in vivo is sufficient to enhance LTP induction in the mouse hippocampal CA1 region, suggesting that activation of MC4R may improve hippocampus-dependent learning and memory.

\section{Discussion}

MC4R is well known to regulate food intake and energy balance within the hypothalamus, but whether this receptor functions in other brain regions has just begun to be unraveled. Detection of prominent MC4R expression in the hippocampus prompted us to speculate that this melanocortin receptor subtype modulates higher cognitive brain functions, such as learning and memory. In this study, we report that MC4R in postsynaptic neurons of the hippocampus is important for both structural and functional synaptic plasticity. Whereas the basal level of MC4R is critical for the maintenance of dendritic spine structure and GluA1 content in the spines, activation of the receptor promotes dendritic spine maturation and enhances surface insertion of GluA1-containing AMPA receptors, revealing the essential role of MC4R in regulating the plasticity of excitatory synapses. Importantly, MC4R is critical for maintaining spine integrity in the CA1 region of hip- 
pocampus, and in vivo delivery of MC4R agonist is sufficient to facilitate LTP in CA1 region. Given that the increase of synaptic strength and LTP are positively associated with learning and memory, our findings have uncovered a new role of MC4R in the hippocampus, revealing a novel molecular link between learning functions and energy metabolism.

Emerging studies have revealed the pivotal roles of various neuromodulators, such as dopamine, serotonin, and norepinephrine, in regulating learning and memory through interaction with their respective subfamilies of GPCRs. Prominent expression of melanocortin peptides and their cognate GPCR MC4R in hippocampal circuit has been reported for nearly two decades (Gantz et al., 1993; Siljee-Wong, 2011), yet the roles of the melanocortin system in hippocampus remained unexplored. Our findings provide the first evidence that activation of MC4R at hippocampal excitatory synapses is critical for the regulation of cellular mechanisms underlying learning and memory. The melanocortin system in hypothalamus is under precise control of various signaling pathways, such as leptin signaling (Shimizu et al., 2007). In particular, leptin increases mRNA level of the $\alpha$-MSH precursor POMC, which in turn enhances production of $\alpha$-MSH (Balthasar et al., 2004). Given that leptin signaling facilitates activitydependent synaptic plasticity and hippocampus-dependent learning and memory (Harvey et al., 2006), it will be of interest to examine whether leptin regulates hippocampal plasticity through modulating MC4R signaling, or whether melanocortin and leptin systems elicit independent and additive effects on learning and memory.

Morphological changes of dendrite spines, together with functional modification of synapses, underlie synaptic plasticity as well as learning and memory (Kozorovitskiy et al., 2005). Strengthening of excitatory synapses is attributed to synaptic accumulation of AMPA receptors and enlargement of dendritic spines. The major postsynaptic signaling mechanisms that underlie synaptic potentiation and spine enlargement include increased surface trafficking and synaptic insertion of AMPA receptor and actin remodeling of spines. Stimulation of MC4R triggers the activation of PKA signaling through coupling of $\mathrm{G} \alpha$ s and adenylyl cyclase (Siljee-Wong, 2011). It is noteworthy that PKA is critical for hippocampus-dependent synaptic plasticity through direct phosphorylation of synaptic proteins or neurotransmitter receptors, such as the AMPA receptor subunit GluA1 (Abel and Nguyen, 2008). In particular, the PKAdependent phosphorylation of GluA1 at Ser 845 is required for synaptic insertion of the receptor, thereby promoting memory formation and consolidation (Monfils et al., 2009). Here, we show that $\mathrm{G} \alpha_{\mathrm{s}}$-cAMP-PKA signaling mediates the action of MC4R on the regulation of neurotransmission and LTP, thus providing the first experimental evidence to support the role of MC4R-PKA signaling in learning and memory. Nonetheless, the detailed mechanisms underlying the coordinated structural and functional changes of the synapses following MC4R activation await further investigation. For example, the PKA-dependent phosphorylation and synaptic incorporation of GluAl may directly trigger the enlargement of dendritic spines (Kopec et al., 2007). Alternatively, PKA signaling regulates dendritic spine morphology through phosphorylation of various candidate actin regulators, such as Rac1 GEF Tiam2, WAVE1, and RhoA (Ethell and Pasquale, 2005). Furthermore, MC4R may also contribute to synaptic plasticity and memory formation through regulating cAMP response element-binding (CREB)-dependent gene transcription (Bito and Takemoto-Kimura, 2003). In this context, it is noteworthy that reduction of MC4R signaling results in down- regulation of $b d n f$ mRNA in the ventromedial hypothalamus, and blockade of activation of BDNF and its cognate receptor TrkB abolishes the action of MC4R in food uptake (Xu et al., 2003). In the hippocampus, dendritic spine remodeling requires BDNF/TrkB signaling (Lai et al., 2012), which can be modulated by cAMP gating (Ji et al., 2005). Given that cAMP-PKAdependent regulation of the $\mathrm{BDNF} / \mathrm{TrkB}$ signaling is involved in various stages of memory formation, such as memory acquisition and consolidation (Yamada and Nabeshima, 2003; Monfils et al., 2007), it will be of interest to examine whether MC4R modulates hippocampal synaptic plasticity, learning, and memory through its effect on the BDNF/TrkB pathway.

The importance of MC4R in hippocampal synaptic plasticity revealed in the present study suggests that alteration of the melanocortin system can result in cognitive dysfunctions. It has been reported that levels of $\alpha-\mathrm{MSH}$ are reduced in the cerebrospinal fluids of patients with dementia of the Alzheimer type and, upon aging, $\alpha$-MSH-positive nerve fibers terminate in the brain areas involved in Alzheimer's disease (AD) (Rainero et al., 1988). In addition, $\alpha$-MSH can protect rat hippocampus from interleukin$1 \beta$-induced memory impairment, possibly through MC4R (Gonzalez et al., 2009). Thus, it will be of interest to further characterize the physiological functions of the melanocortin system in hippocampus, in particular by dissecting the role of MC4R in learning and memory, as well as in the progression of cognitive deficits in $\mathrm{AD}$. It is tempting to speculate that pharmacological agents that can increase $\alpha$-MSH level or enhance activation of MC4R can potentially be developed into therapeutic strategies for cognitive deficits associated with aging or $\mathrm{AD}$.

\section{References}

Abel T, Nguyen PV (2008) Regulation of hippocampus-dependent memory by cyclic AMP-dependent protein kinase. In: Progress in brain research, Chap 6 (Sossin WS, Lacalle JC, Castellucci VF, Belleville S, eds), pp 97115. Amsterdam: Elsevier.

Balthasar N, Coppari R, McMinn J, Liu SM, Lee CE, Tang V, Kenny CD, McGovern RA, Chua SC Jr, Elmquist JK, Lowell BB (2004) Leptin receptor signaling in POMC neurons is required for normal body weight homeostasis. Neuron 42:983-991. CrossRef Medline

Bito H, Takemoto-Kimura S (2003) Ca(2+)/CREB/CBP-dependent gene regulation: a shared mechanism critical in long-term synaptic plasticity and neuronal survival. Cell Calcium 34:425-430. CrossRef Medline

Cheung ZH, Ip NY (2011) From understanding synaptic plasticity to the development of cognitive enhancers. Int J Neuropsychopharmacol 14: 1247-1256. CrossRef Medline

Chklovskii DB (2004) Synaptic connectivity and neuronal morphology: two sides of the same coin. Neuron 43:609-617. CrossRef Medline

Datta PC, King MG (1982) $\alpha$-Melanocyte-stimulating hormone and behavior. Neurosci Biobehav Rev 6:297-310. CrossRef Medline

Derkach VA, Oh MC, Guire ES, Soderling TR (2007) Regulatory mechanisms of AMPA receptors in synaptic plasticity. Nat Rev Neurosci 8:101113. CrossRef Medline

Earnshaw BA, Bressloff PC (2006) Biophysical model of AMPA receptor trafficking and its regulation during long-term potentiation/long-term depression. J Neurosci 26:12362-12373. CrossRef Medline

Esteban JA, Shi SH, Wilson C, Nuriya M, Huganir RL, Malinow R (2003) PKA phosphorylation of AMPA receptor subunits controls synaptic trafficking underlying plasticity. Nat Neurosci 6:136-143. CrossRef Medline

Ethell IM, Pasquale EB (2005) Molecular mechanisms of dendritic spine development and remodeling. Prog Neurobiol 75:161-205. CrossRef Medline

Fu AK, Hung KW, Fu WY, Shen C, Chen Y, Xia J, Lai KO, Ip NY (2011) APC(Cdh1) mediates EphA4-dependent downregulation of AMPA receptors in homeostatic plasticity. Nat Neurosci 14:181-189. CrossRef Medline

Fu W-Y, Chen Y, Sahin M, Zhao X-S, Shi L, Bikoff JB, Lai K-O, Yung W-H, Fu AKY, Greenberg ME, Ip NY (2007) Cdk5 regulates EphA4-mediated 
dendritic spine retraction through an ephexin1-dependent mechanism. Nat Neurosci 10:67-76. CrossRef Medline

Gantz I, Miwa H, Konda Y, Shimoto Y, Tashiro T, Watson SJ, DelValle J, Yamada T (1993) Molecular cloning, expression, and gene localization of a fourth melanocortin receptor. J Biol Chem 268:15174-15179. Medline

Garfield AS, Lam DD, Marston OJ, Przydzial MJ, Heisler LK (2009) Role of central melanocortin pathways in energy homeostasis. Trends Endocrinol Metab 20:203-215. CrossRef Medline

Gómez-Pinilla F (2008) Brain foods: the effects of nutrients on brain function. Nat Rev Neurosci 9:568-578. CrossRef Medline

Gonzalez PV, Schiöth HB, Lasaga M, Scimonelli TN (2009) Memory impairment induced by IL- 1 beta is reversed by alpha-MSH through central melanocortin-4 receptors. Brain Behav Immun 23:817-822. CrossRef Medline

Harvey J, Solovyova N, Irving A (2006) Leptin and its role in hippocampal synaptic plasticity. Prog Lipid Res 45:369-378. CrossRef Medline

Ji Y, Pang PT, Feng L, Lu B (2005) Cyclic AMP controls BDNF-induced TrkB phosphorylation and dendritic spine formation in mature hippocampal neurons. Nat Neurosci 8:164-172. CrossRef Medline

Kopec CD, Li B, Wei W, Boehm J, Malinow R (2006) Glutamate receptor exocytosis and spine enlargement during chemically induced long-term potentiation. J Neurosci 26:2000-2009. CrossRef Medline

Kopec CD, Real E, Kessels HW, Malinow R (2007) GluR1 links structural and functional plasticity at excitatory synapses. J Neurosci 27:1370613718. CrossRef Medline

Kozorovitskiy Y, Gross CG, Kopil C, Battaglia L, McBreen M, Stranahan AM, Gould E (2005) Experience induces structural and biochemical changes in the adult primate brain. Proc Natl Acad Sci U S A 102:17478-17482. CrossRef Medline

Lai KO, Wong AS, Cheung MC, Xu P, Liang Z, Lok KC, Xie H, Palko ME, Yung WH, Tessarollo L, Cheung ZH, Ip NY (2012) Serine phosphorylation of TrkB by Cdk5 is required for activity-dependent structural plasticity and spatial memory. Nat Neurosci 15:1506-1515. CrossRef Medline

Lee YS, Silva AJ (2009) The molecular and cellular biology of enhanced cognition. Nat Rev Neurosci 10:126-140. CrossRef Medline

Liu H, Kishi T, Roseberry AG, Cai X, Lee CE, Montez JM, Friedman JM, Elmquist JK (2003) Transgenic mice expressing green fluorescent protein under the control of the melanocortin-4 receptor promoter. J Neurosci 23:7143-7154. Medline

Man H-Y, Sekine-Aizawa Y, Huganir RL (2007) Regulation of $\alpha$-amino-3hydroxy-5-methyl-4-isoxazolepropionic acid receptor trafficking through PKA phosphorylation of the Glu receptor 1 subunit. Proc Natl Acad Sci U S A 104:3579-3584. CrossRef Medline

McLay RN, Pan W, Kastin AJ (2001) Effects of peptides on animal and human behavior: a review of studies published in the first twenty years of the journal Peptides. Peptides 22:2181-2255. CrossRef Medline
Monfils MH, Cowansage KK, LeDoux JE (2007) Brain-derived neurotrophic factor: linking fear learning to memory consolidation. Mol Pharmacol 72:235-237. CrossRef Medline

Monfils MH, Cowansage KK, Klann E, LeDoux JE (2009) Extinctionreconsolidation boundaries: key to persistent attenuation of fear memories. Science 324:951-955. CrossRef Medline

Murchison CF, Zhang XY, Zhang WP, Ouyang M, Lee A, Thomas SA (2004) A distinct role for norepinephrine in memory retrieval. Cell 117:131-143. CrossRef Medline

O’Dell TJ, Connor SA, Gelinas JN, Nguyen PV (2010) Viagra for your synapses: enhancement of hippocampal long-term potentiation by activation of beta-adrenergic receptors. Cell Signal 22:728-736. CrossRef Medline

Oosterom J, Garner KM, den Dekker WK, Nijenhuis WAJ, Gispen WH, Burbach JPH, Barsh GS, Adan RAH (2001) Common requirements for melanocortin-4 receptor selectivity of structurally unrelated melanocortin agonist and endogenous antagonist, agouti protein. J Biol Chem 276: 931-936. CrossRef Medline

Rainero I, May C, Kaye JA, Friedland RP, Rapoport SI (1988) CSF alphaMSH in dementia of the Alzheimer type. Neurology 38:1281-1284. CrossRef Medline

Schratt GM, Tuebing F, Nigh EA, Kane CG, Sabatini ME, Kiebler M, Greenberg ME (2006) A brain-specific microRNA regulates dendritic spine development. Nature 439:283-289. CrossRef Medline

Segal M (2005) Dendritic spines and long-term plasticity. Nat Rev Neurosci 6:277-284. CrossRef Medline

Shimizu H, Inoue K, Mori M (2007) The leptin-dependent and -independent melanocortin signaling system: regulation of feeding and energy expenditure. J Endocrinol 193:1-9. CrossRef Medline

Siljee-Wong JE (2011) Melanocortin MC(4) receptor expression sites and local function. Eur J Pharmacol 660:234-240. CrossRef Medline

Waltereit R, Weller M (2003) Signaling from cAMP/PKA to MAPK and synaptic plasticity. Mol Neurobiol 27:99-106. CrossRef Medline

Xu B, Goulding EH, Zang K, Cepoi D, Cone RD, Jones KR, Tecott LH, Reichardt LF (2003) Brain-derived neurotrophic factor regulates energy balance downstream of melanocortin-4 receptor. Nat Neurosci 6:736742. CrossRef Medline

Yamada K, Nabeshima T (2003) Brain-derived neurotrophic factor/TrkB signaling in memory processes. J Pharmacol Sci 91:267-270. CrossRef Medline

Yang SN (2000) Sustained enhancement of AMPA receptor- and NMDA receptor-mediated currents induced by dopamine D1/D5 receptor activation in the hippocampus: an essential role of postsynaptic $\mathrm{Ca}^{2+}$. Hippocampus 10:57-63. CrossRef Medline

Yuste R, Bonhoeffer T (2001) Morphological changes in dendritic spines associated with long-term synaptic plasticity. Annu Rev Neurosci 24: 1071-1089. CrossRef Medline 